\title{
Protective Effects of 18$\beta$-Glycyrrhetinic Acid on Monocrotaline-Induced Pulmonary Arterial Hypertension in Rats
}

\begin{abstract}
Min Zhang ${ }^{1 \dagger}$, Zhi Chang ${ }^{1 \dagger}$, Fang Zhao ${ }^{2 \dagger}$, Peng Zhang ${ }^{2}$, Yin-Ju Hao ${ }^{1}$, Lin Yan ${ }^{2}$, Ning Liu ${ }^{3}$, Jun-Li Wang ${ }^{4}$, Lei Bo ${ }^{4}$, Ping $\mathrm{Ma}^{2}$, Wei Zhou ${ }^{2}$, Xuan Ma ${ }^{2}$, Qing-Bin $\mathrm{Xu}^{2 *}$ and Ru Zhou ${ }^{1,3,5 *}$

'Department of Pharmacology, College of Pharmacy, Ningxia Medical University, Yinchuan, China, ${ }^{2}$ General Hospital of Ningxia Medical University, Yinchuan, China, ${ }^{3}$ Ningxia Hui Medicine Modern Engineering Research Center and Collaborative Innovation Center, Ningxia Medical University, Yinchuan, China, ${ }^{4}$ Foreign Language Teaching Department, Ningxia Medical University, Yinchuan, China, ${ }^{5}$ Key Laboratory of Hui Ethnic Medicine Modernization, Ministry of Education, Ningxia Medical University, Yinchuan, China
\end{abstract}

\section{OPEN ACCESS}

Edited by:

Zhi-Cheng Jing,

Fuwai Hospital (CAMS), China

Reviewed by:

Zhiyu Dai,

Northwestern University,

United States

Xiao-Qing Sun,

VU University Medical Center,

Netherlands

${ }^{*}$ Correspondence:

Qing-Bin Xu

505103783@qq.com

Ru Zhou

zhouru@nxmu.edu.cn

${ }^{\dagger}$ These authors have contributed equally to this work

Specialty section:

This article was submitted to Respiratory Pharmacology,

a section of the journal

Frontiers in Pharmacology

Received: 02 November 2018 Accepted: 07 January 2019

Published: 22 January 2019

Citation:

Zhang M, Chang Z, Zhao F

Zhang P, Hao Y-J, Yan L, Liu N, Wang J-L, Bo L, Ma P, Zhou W, Ma X, $X U \mathrm{Q}-B$ and Zhou $R$ (2019) Protective Effects of 18 $\beta$-Glycyrrhetinic Acid on Monocrotaline-Induced Pulmonary

Arterial Hypertension in Rats.

Front. Pharmacol. 10:13

doi: 10.3389/fphar.2019.00013
Pulmonary arterial hypertension $(\mathrm{PAH})$ is a destructive and rare disorder characterized by a progressive increase in pulmonary artery pressure and vasoconstriction, ultimately

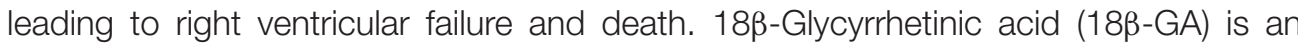
active ingredient in the commonly used Chinese herbal medicine radix glycyrrhizae, and it possesses antioxidant, anti-inflammatory, anti-tumor, and other pharmacological properties. This study aimed to determine whether 18 $\beta$-GA has protective effects against monocrotaline (MCT)-induced PAH and whether it is associated with oxidative stress. The PAH of rats was induced by MCT $(60 \mathrm{mg} / \mathrm{kg})$ and oral administration of $18 \beta$-GA (100, 50, or $25 \mathrm{mg} / \mathrm{kg} /$ day), sildenafil (30 mg/kg), or saline for 21 consecutive days. The development of PAH was evaluated by hemodynamic parameters and right ventricular hypertrophy index. Hematoxylin and eosin staining, Masson trichrome staining, and electron microscopy were used to determine the degree of vascular remodeling and proliferation in lung tissue. Moreover, the antioxidant capacity and malondialdehyde levels in the lungs were measured according to the instructions provided by the test kits, and the expression levels of nicotinamide adenine dinucleotide phosphate oxidase-2 (Nox2) and Nox4 were detected through Western blot analysis. Results of our study indicated that $18 \beta-G A$ treatment significantly improved the hemodynamic and pathomorphological data of the rats, reduced the changes in oxidative stress biomarkers, and inhibited Nox2 and Nox4 expression. Our research indicated that $18 \beta$-GA has a protective effect against MCT-induced PAH by inhibiting oxidative stress in rats.

Keywords: pulmonary arterial hypertension, monocrotaline, $18 \beta$-glycyrrhetinic acid, oxidative stress, nicotinamide adenine dinucleotide phosphate oxidase

\section{INTRODUCTION}

Pulmonary arterial hypertension $(\mathrm{PAH})$ is a severe and multifactorial cardiovascular syndrome that restricts flow through pulmonary arterial circulation, and it is characterized by pulmonary arterial pressure of $\geq 25 \mathrm{mmHg}$ at rest and $\geq 30 \mathrm{mmHg}$ when moving. It is distinguished by resistance of pulmonary arteries and an increase in pulmonary arterial pressure, which trigger subsequent right 
heart hypertrophy and failure and ultimately result in death (Wu et al., 2016; Gao et al., 2017; Lan et al., 2018). At present, eventful progress has been made in the comprehension of pathogenesis and treatment of PAH. However, the disease remains incurable, which is related to its relatively high morbidity and mortality. The 5- and 7-year survival rates of PAH patients are 57 and 49\%, respectively (Thenappan et al., 2010; Benza et al., 2012).

The pathological mechanism of $\mathrm{PAH}$ is complex, involving the interaction of multiple factors. The pathogenesis of $\mathrm{PAH}$ includes vascular remodeling, vasoconstriction, and thrombosis, where vascular remodeling plays a critical step in the progression of PAH. Studies have reported that oxidative stress is the key mechanism leading to vascular remodeling in $\mathrm{PAH}$ (Demarco et al., 2010), and this condition is triggered by nicotinamide adenine dinucleotide phosphate oxidase (Nox). In PAH, Nox2, and Nox4 are up-regulated (Green et al., 2012; Wedgwood et al., 2013) and implicated in the development of increased pulmonary artery resistance and pressure (Barman et al., 2014; Wu et al., 2017a). Therefore, the antioxidation and interference of Nox may be considered therapeutic targets of PAH inhibition. Nevertheless, the conventional pharmaceutical therapies for $\mathrm{PAH}$, including prostacyclin analogs, phosphodiesterase type-5 enzyme (PED-5) inhibitors, and endothelin receptor antagonists (Aiello et al., 2016), are unsatisfactory because of their toxic side effects and limited effects on the inhibition of disease progression (Humbert et al., 2010). Therefore, the discovery and development of safe and effective agents to prevent or delay the progression of PAH in the field of traditional medicine are of critical clinical significance (Galiè and Manes, 2013).

Chinese herbs have been widely investigated because of their abundant resources, safe use, and multitargeted efficacy. As a special medicinal material in Ningxia, China, radix glycyrrhizae (leguminosae) is a traditional Chinese herbal medicine that likely originated from Shennong (a specialist of herbs in ancient China) herbs. It has been widely used in the practice of traditional Chinese medicines for centuries (Chung et al., 2001; Yang et al., 2014; Young et al., 2016). Radix glycyrrhizae contains the major bioactive triterpene glycoside $18 \beta$-glycyrrhetinic acid (18 $\beta$-GA), which possesses multiple pharmacological activities, such as antioxidant, anticancer, and anti-inflammation activities (Hasan et al., 2014) (Figure 1). The biological effects of $18 \beta-G A$ in improving lung function have been demonstrated under various conditions in animals; for example, $18 \beta$-GA alleviates radiationinduced lung injury in mice and lung fibrosis by inhibiting inflammation (Tzuchien et al., 2010; Chen et al., 2017). 18 $\beta$-GA effectively suppresses the proliferation of non-small cell lung cancer (Huang et al., 2013). All of these studies have reflected that $18 \beta$-GA has beneficial effects on lungs. However, its protective effects on PAH remain to be investigated.

On the basis of these considerations, we hypothesized that $18 \beta$-GA can improve PAH. To confirm this hypothesis, we established a PAH model of rats by single subcutaneous injection of MCT for 21 days and used 18 $\beta$-GA for the therapy of rats with MCT-induced PAH (Mcmurtry et al., 2007; Wu et al., 2017a,b). Our current research aimed to examine the protective effect of $18 \beta-\mathrm{GA}$ on PAH induced by MCT and further explore its antioxidant effects.

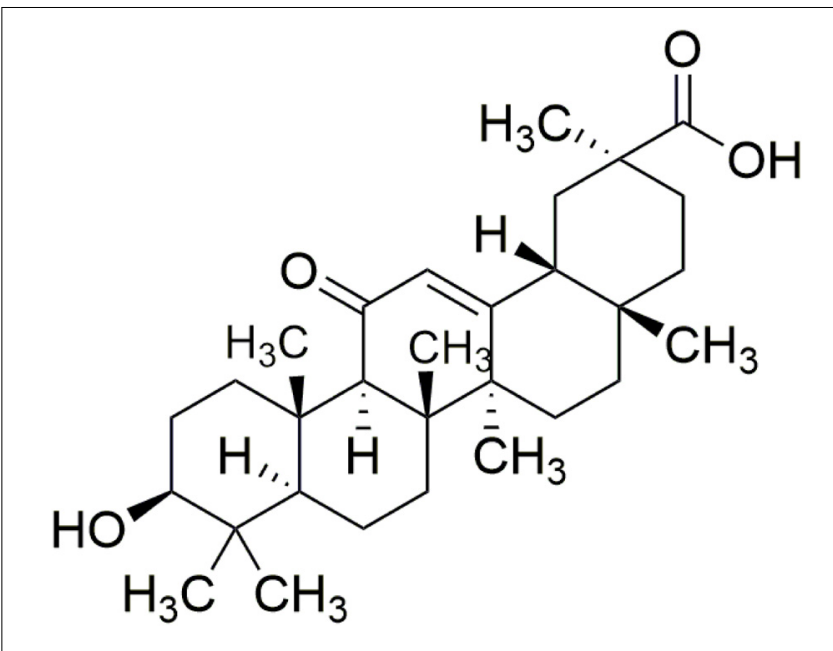

FIGURE 1 | Structure of $18 \beta$-glycyrrhetinic acid. The molecular formula for $18 \beta$-glycyrrhetinic acid is $\mathrm{C} 3 \mathrm{OH} 46 \mathrm{O} 4$, and the molecular weight is 470.68 .

\section{MATERIALS AND METHODS}

\section{Animals}

Male Sprague-Dawley rats (220-260 g, 7 weeks old) were supplied by the animal experimental center of Ningxia Medical University (SYXK Ningxia 2015-0001). The animal experimental procedures were approved in accordance with the Institutional Animal Care and Use Committee of Ningxia Medical University. All the rats were housed under specific conditions ( $12 \mathrm{~h}$ light $/ 12 \mathrm{~h}$ dark cycle, $22 \pm 3^{\circ} \mathrm{C}$ ) and given free access to water and food.

\section{Reagents}

$18 \beta$-GA with purity of greater than $99.3 \%$ revealed by HPLC analysis was purchased from Yuan Ye Biotechnology Co., Ltd. (Shanghai, China, Cat NO. F20O8J46129). MCT was provided by Sigma (St. Louis, MO, United States). Anti- $\beta$-actin (Cat NO. 20536-1-AP) polyclonal antibodies were provided by Proteintech Group (CA, United States). Anti-NADPH oxidase 4 antibody and anti-Nox-2/gp91phox antibody were purchased from ABCam Biotechnology (Cat NO. 14347-1-AP, 190131-AP). Determination kits for superoxide dismutase (SOD) (Cat NO. A001-1), malonyldialdehyde (MDA) (Cat NO. A0031), glutathione peroxidase (GSH-PX) (Cat NO. A005), total antioxidant capacity (T-AOC) (Cat NO. A015), and catalase (CAT) (Cat NO. A007-1) were procured from Nanjing Jiancheng Research Institute of Biotechnology (Jiangsu, China).

\section{Experimental Design}

One hundred and twenty male rats were randomly assigned to six groups: control group (normal control, $n=20$ ), model group (MCT exposure, $n=20$ ), sildenafil group (MCT+sildenafil $30 \mathrm{mg} / \mathrm{kg} / \mathrm{day}, n=20$ ), and $18 \beta$-GA groups (MCT+18 $\beta$-GA 100, 50 , and $25 \mathrm{mg} / \mathrm{kg} / \mathrm{day}, n=20)$. MCT $(60 \mathrm{mg} / \mathrm{kg})$ was administered to induce PAH by single abdominal subcutaneous injection. The control group simultaneously received normal saline on day 0 . Subsequently, $18 \beta-\mathrm{GA}(100,50$, and $25 \mathrm{mg} / \mathrm{kg} / \mathrm{day}$, dissolved in 
saline) or sildenafil (30 mg/kg/day, suspended in saline) (Wu et al., 2017a,b) was intragastrically administered once daily from days 21 to 42 . The same volume of physiological saline was given to the control and MCT-exposed groups. The Figure 2 illustrates the experimental design, including induction of $\mathrm{PAH}$, subsequent administration time, and other experimental analyses.

\section{Survival Analysis}

Survival was assessed over the entire experimental period from days 1 to 42, during which the effect of $18 \beta$-GA on survival rate of MCT-injected rats was examined. Survival curves were compared using Kaplan-Meier analysis.

\section{Hemodynamic Measurements}

The rats were anesthetized by intraperitoneal injecting $20 \%$ urethane $(1 \mathrm{ml} / 100 \mathrm{~g})$, and their pressure was measured. Following stable anesthesia, the rats were placed on an operating table in a supine position. A heparin-filled polyethylene catheter was inserted into the right ventricle through the right external jugular vein to detect the mean pulmonary arterial pressure (mPAP) and right ventricular systolic pressure (RVSP) via an MPA-cardiac function acquisition analysis system (Alcott Biotech, Shanghai, China).

\section{Evaluation of Right Ventricular Hypertrophy}

Following the pressure measurements, the rats were sacrificed, and the lungs and hearts were obtained. The lung was separated into two parts for histopathological examination and protein assay. The hearts were divided into the right ventricle (RV) and left ventricle (LV) plus the inter-ventricular septum (S). The ratio of the weight of the RV to the LV plus $S[R V /(L V+S)]$ was calculated as the right ventricular hypertrophy index (RVHI).

\section{Histomorphometric Analysis}

\section{H\&E Staining and Masson Trichrome Staining}

The rats were sacrificed by cervical dislocation under anesthesia following hemodynamic measurements. The isolated lower lobe of the left lung tissue was rinsed with physiological saline and fixed with $4 \%$ paraformaldehyde for $48 \mathrm{~h}$ for morphometric analysis. After $48 \mathrm{~h}$ of fixation, the lung tissues were embedded in paraffin, cut into $4 \mu \mathrm{m}$-thick sections, and subjected to hematoxylin and eosin (H.E) staining and Masson trichrome staining. The structures of the pulmonary arteries and the degree of fibrosis in the artery wall in the lungs were remodeled and examined through microscopic assessment. Twenty small pulmonary vessels with diameters of 50-300 $\mu \mathrm{m}$ were randomly selected from each section and analyzed at a magnification of $400 \times$. Two indices reflecting pulmonary arterial remodeling were calculated as follows: (1) ratio of pulmonary arterial wall thickness $(\mathrm{WT} \%)=100 \% \times($ external diameter - internal diameter)/external diameter and (2) ratio of pulmonary arterial wall area $(\mathrm{WA} \%)=100 \% \times($ transection area of the walls-lumen area)/transection area of the walls.

\section{Morphological Evaluation by the Electron Microscope}

After right heart catheterization of pressure measurements, a sample of the left lung tissues was collected, fixed for $2 \mathrm{~h}$ in Bouin's fixative at $4^{\circ} \mathrm{C}$, separated into $1 \mathrm{~mm} \times 1 \mathrm{~mm} \times 1 \mathrm{~mm}$ cubes, rinsed three times with phosphate buffer, immersed in $2 \%$ osmium tetroxide, dehydrated with an alcohol gradient, and embedded. Ultrathin $75 \mathrm{~nm}$-thick sections were collected and stained with uranyl acetate and lead citrate. In the sections of lung tissues, the histopathological changes were investigated under electron microscopy (Olympus, Tokyo, Japan, 6,000×), and images were randomly obtained in a blinded manner (Karpuz et al., 2017; Shi G.J. et al., 2017; Zhang et al., 2017).

\section{Measurements of Oxidative Stress}

All the rats were sacrificed after hemodynamic measurements. The prepared lung tissues were homogenized in ice-cold saline $(0.9 \%)$ in glass homogenizer. Then the homogenate was centrifuged at $2500 \mathrm{r} / \mathrm{min}$ for $10 \mathrm{~min}$ at $4^{\circ} \mathrm{C}$ temperature. Supernatant of homogenate $(10 \%, \mathrm{w} / \mathrm{v})$ was employed for the subsequent tests. The activities of SOD, CAT, MDA, GSH$\mathrm{PX}$, and T-AOC in the supernatant was determined according to the instructions of the respective testing kit (Nanjing Jiancheng Bioengineering Research Institute, Jiangsu, China) and a microplate reader (1510, Thermo Fisher, United States).

\section{Western Blot Analysis}

As described previously, the lung tissue samples from all of the groups were homogenized in lysis buffer at $4^{\circ} \mathrm{C}$. The soluble proteins of Nox 2 and Nox 4 were collected and centrifuged at $12,000 \mathrm{rpm}$ for $20 \mathrm{~min}$ at $4^{\circ} \mathrm{C}$. The total protein concentration was measured using the BCA protein assay kit (Beyotime, Jiangsu, China). The equivalent amounts of protein lysates in

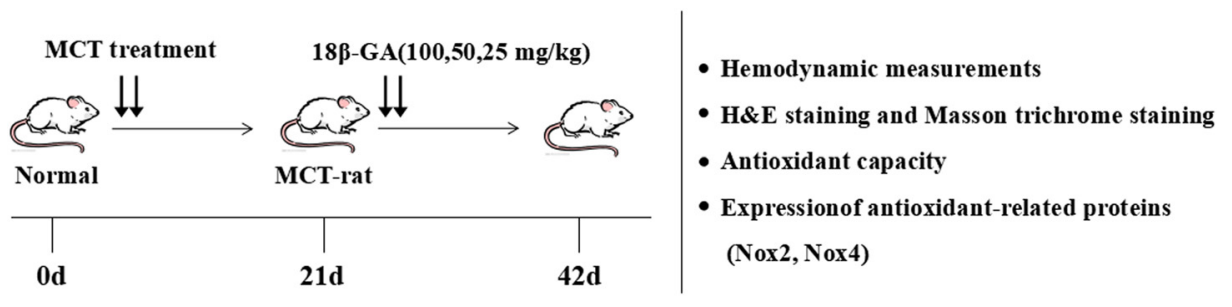

FIGURE 2 | Experimental design. PAH model of rats was established by single subcutaneous injection of MCT (60 mg/kg). After 21 days, 18 3 -GA (100, 50, and $25 \mathrm{mg} / \mathrm{kg} /$ day) was intragastrically administered. At the end of the treatment, lung structure and function were evaluated through various experimental methods. 
each group were separated by $8 \%$ sodium dodecyl sulfatepolyacrylamide gel electrophoresis (SDS-PAGE), and the proteins on the gel were subsequently transferred onto a nitrocellulose membrane $(200 \mathrm{~mA}, 2 \mathrm{~h})$. The membranes were blocked with blocking PBST (PBS involving $0.1 \%$ Tween-20) buffer containing $5 \%$ skim milk at room temperature for $2 \mathrm{~h}$ and then probed with primary rabbit monoclonal anti-Nox-2 antibody (1:3000; ABCam Biotechnology, CA, United States) and rabbit monoclonal anti-Nox-4 antibody (1:1500; ABCam Biotechnology, CA, United States) overnight at $4^{\circ} \mathrm{C}$. Subsequently, the membranes were washed with PBST and incubated with goat anti-rabbit IgG antibody (1:3000; Proteintech Group, United States) for $2 \mathrm{~h}$ at room temperature. The anti- $\beta$-actin monoclonal antibody (1:1000; Proteintech Group, United States) served as a control. Membranes were washed three times in PBST, and the protein bands were visualized with enhanced chemiluminescent reagents (Applygen technology, Beijing, China). The grayscale values of each band on the blots were analyzed with Quantitative One (Bio-Rad Company, CA, United States).

\section{Statistical Analysis}

Experimental data were analyzed with SPSS software 19.0 (SPSS Inc., Chicago, IL, United States) and plotted as the mean \pm standard error of the mean (SEM). Data were examined through one-way ANOVA and Student's Newman-Keuls test for post hoc test to determine differences between the control and experimental groups. In all of the statistical tests, $p<0.05$ was defined statistically significant.

\section{RESULTS}

\section{Effects of 18 $\beta-$ GA on the Survival of Rats}

After MCT was injected, PAH developed and continued to cause the deterioration of the rats until their deaths. The rats' survival rate was assessed in the entire experimental period of 42 days. The effects of $18 \beta-G A$ on the survival rate of different groups of rats were also examined in the same period. At the end of our experiment, no deaths were observed in the control group, whereas the survival rates of the $18 \beta$-GA-treated group $(100 \mathrm{mg} / \mathrm{kg})$ and sildenafil group were significantly higher than that of the PAH group ( $p<0.05$; Figure 3).

\section{Effects of 18 $\beta$-GA on Hemodynamic Measurement}

We assessed the protective effect of $18 \beta-$ GA on MCT-induced PAH by detecting the levels of mPAP and RVSP. At 6 weeks after MCT was administered, MPAP and RVSP levels significantly increased in the MCT group compared with that in the control group ( $p<0.01$; Figure 4 ), and this result demonstrated the successful establishment of the MCT-induced rat model. We started treatment intervention with $18 \beta$-GA and sildenafil at 3 weeks after MCT exposure. The rats treated with $18 \beta-\mathrm{GA}$ (100 and $50 \mathrm{mg} / \mathrm{kg}$ ) and sildenafil $(30 \mathrm{mg} / \mathrm{kg}$ ) demonstrated significantly lower mPAP and RVSP levels than the MCT group $(p<0.01$ and $p<0.05$, respectively; Figure 4). All these results

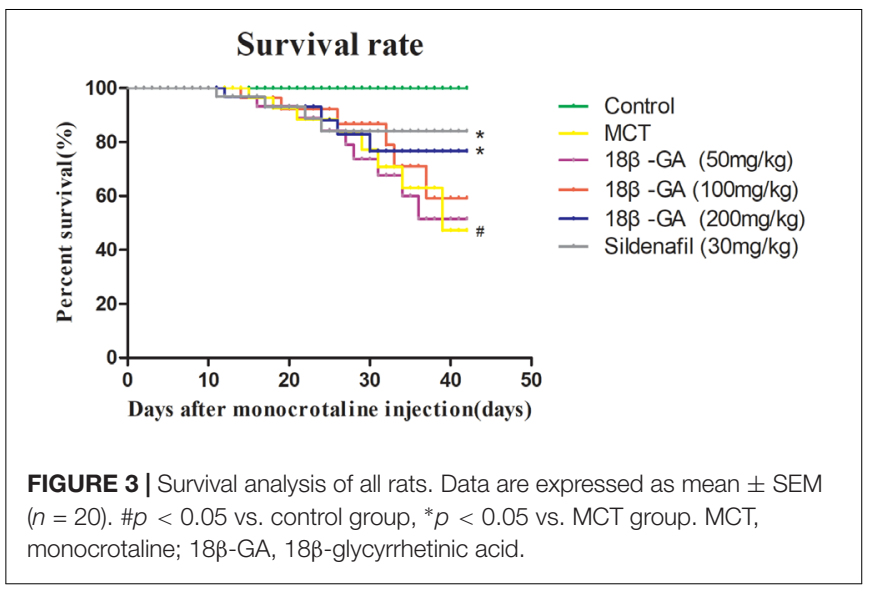

demonstrated that oral administration of $18 \beta$-GA could alleviate MCT-induced $\mathrm{PAH}$ in rats.

\section{Effects of 18 $\beta$-GA on Right Heart Hypertrophy}

The weight ratio of $\mathrm{RV} / \mathrm{LV}+\mathrm{S}$ was calculated to assess the extent of right ventricular hypertrophy $(\mathrm{RVH})$. A significant increase in RVHI was observed due to an increase in pulmonary arterial pressure. Changes in this ratio further confirmed that severe $\mathrm{PAH}$ was induced after MCT was subcutaneously injected. In the MCT-treated group, RVHI significantly increased $(p<0.01$ and $p<0.05$; Figure 5). In comparison with RVHI in the MCT group, RVHI could be markedly decreased by two doses of $18 \beta$-GA (100 and $50 \mathrm{mg} / \mathrm{kg})$ and sildenafil $(30 \mathrm{mg} / \mathrm{kg})(p<0.01$ and $p<0.05$, respectively; Figure 5). As expected, $18 \beta-\mathrm{GA}$ obviously alleviated MCT-induced RVH.

\section{Effects of 18 $\beta$-GA on Pulmonary Vascular Remodeling in MCT Induced PAH H\&E Staining and Masson Trichrome Staining}

Pulmonary vascular remodeling is one of the crucial causes leading to PAH development. We performed hematoxylin and eosin (H\&E) staining and Masson trichrome staining to evaluate the pathological changes in the small pulmonary arteries (50$200 \mu \mathrm{m})$. H\&E staining demonstrated that the pulmonary artery of the control group was characterized by a thin-walled medial wall and a large lumen (Figure 6A). In comparison with the lung histology of the control group, the MCT group demonstrated increased pulmonary arterial wall thickness and luminal stenosis, as well as excessive inflammatory cell count. After the rats were treated with $18 \beta-$ GA and sildenafil, the changes in pulmonary arterial morphology significantly improved. In addition, pulmonary parietal wall thickness indices (WA\% and WT\%) were determined to assess the effect of $18 \beta-\mathrm{GA}$ on pulmonary vascular remodeling. WA\% and WT\% remarkably increased in the MCT-treated group compared with those in the control group ( $p<0.01$ and $p<0.05$; Figure 6A). In the $18 \beta-\mathrm{GA}$ (100 and $\mathrm{pmg} \mathrm{mg} / \mathrm{kg}$ ) and sildenafil $(30 \mathrm{mg} / \mathrm{kg})$ groups, WA\% and WT\% decreased compared with the MCT group ( $p<0.01$ and $p<0.05$, respectively; Figure $6 \mathrm{C}$ ). In 
A
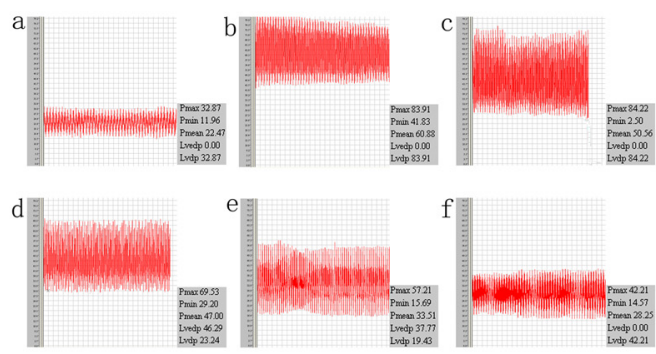

B

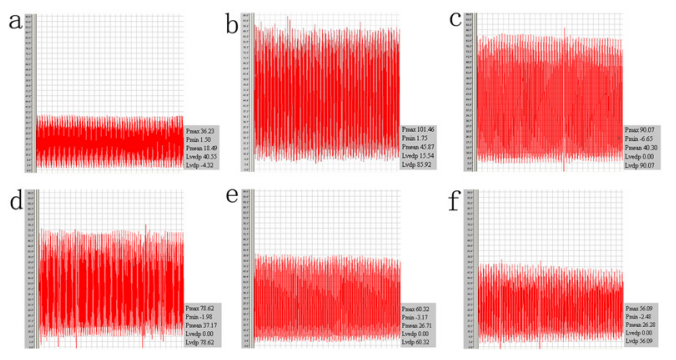

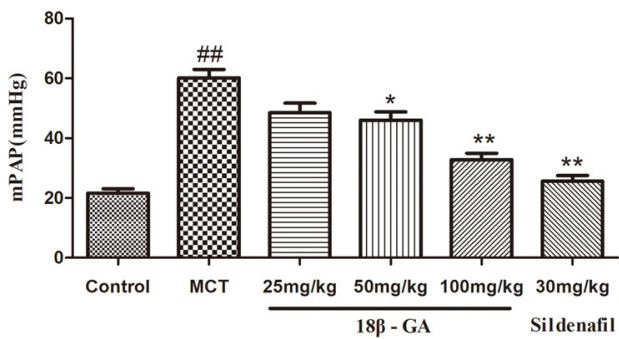

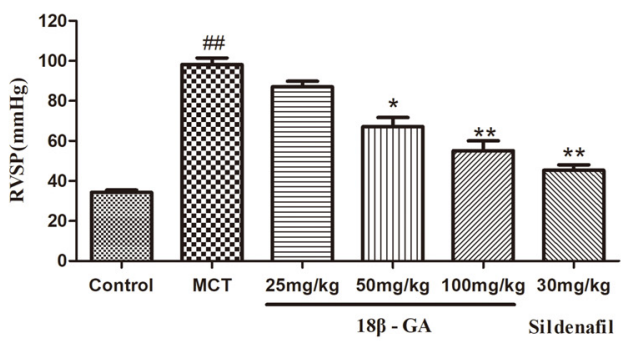

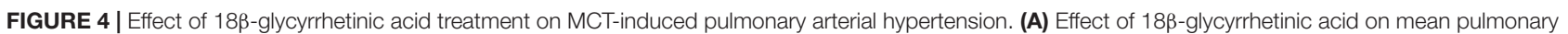

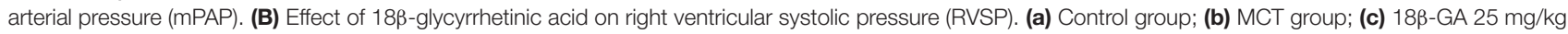
group; (d) $18 \beta$-GA 50 mg/kg group; (e) $18 \beta-G A 100 \mathrm{mg} / \mathrm{kg}$ group; (f) Sildenafil $30 \mathrm{mg} / \mathrm{kg}$ group. Data are expressed as mean \pm SEM $(n=10)$. \#\# $p<0.01 \mathrm{vs}$. control group, ${ }^{*} p<0.05,{ }^{* *} p<0.01$ vs. MCT group. MCT, monocrotaline; $18 \beta$-GA, $18 \beta$-glycyrrhetinic acid; mPAP, mean pulmonary arterial pressure; RVSP, right ventricular systolic pressure.

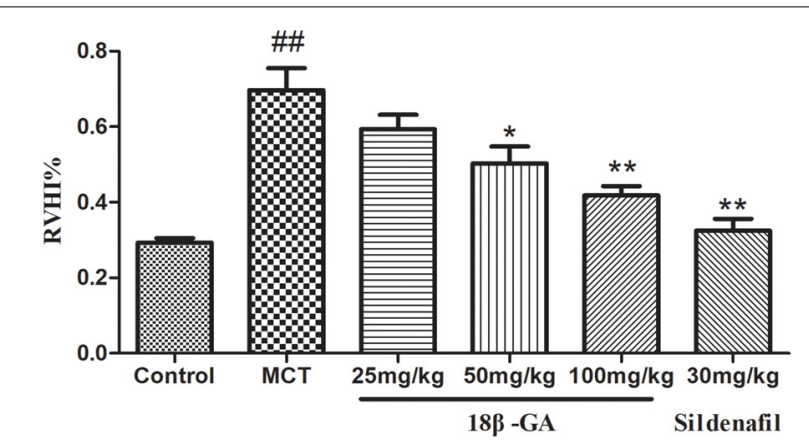

FIGURE 5 | Effect of $18 \beta$-glycyrrhetinic acid on right ventricular hypertrophy index (RVHI). Data are expressed as mean \pm SEM $(n=10)$. $\# \# p<0.01$ vs. control group, ${ }^{*} p<0.05,{ }^{* *} p<0.01$ vs. MCT group. MCT, monocrotaline 18$\beta$-GA, $18 \beta$-glycyrrhetinic acid; RVHI, right ventricular hypertrophy index.

the control group, Masson staining displayed a small amount of collagen fibers in the field of vision (Figure 6B), but the MCT group demonstrated many disordered proliferating collagen fibers in the blood vessel wall and surrounding tissues (Figure 6B). Similar to the sildenafil-treated rats, 18 $\beta$-GAtreated rats showed a significant improvement in collagen fiber proliferation (Figure 6B). These results indicated that $18 \beta$-GA administration significantly improved the pathological changes in the pulmonary vasculature induced by MCT.

\section{Electron Microscopy Analyses}

Changes in pulmonary artery ultrastructure were analyzed by transmission electron microscopy. Under the electron microscope, the pulmonary vascular of the rats in the control group displayed normal morphological characteristics. Endothelial cells, smooth muscle cells, and connective tissue components were also detected as normal. In the MCT group, the endothelial cells in the middle and small muscles of the lungs were hyperplastic, hypertrophic, and swollen; the smooth muscle cells were hypertrophic. The elastic membrane structure was incomplete and fractured. A large number of mitochondria and endoplasmic reticuli were observed in the cytoplasm. After treatment with $18 \beta-G A$ and sildenafil, the endothelial cells of the middle and small muscular arteries in the rats were flat, the vacuoles in the cytoplasm significantly decreased, the elastic membrane structure was clear, and the ultrastructural changes in the pulmonary artery significantly improved compared with those in the model group. These results suggested that the histopathological features of the lung tissues of the rats in the $18 \beta$-GA-treated groups were alleviated (Figure 7).

\section{Effects of 18 $\beta$-GA on Oxidative Stress}

The biomarker levels of oxidative stress in lung tissues were measured to demonstrate the effects of $18 \beta$-GA on MCT-induced oxidative stress. In comparison with the levels in the control group, the SOD, CAT, T-AOC, and GSH-PX levels in the MCTexposed group markedly decreased, whereas their MDA levels significantly increased $(p<0.01$ and $p<0.05$, respectively; Figure 8). All these results implied that MCT could aggravate oxidative stress. Subsequently, administration of 18ß-GA downregulated the MDA level and increased SOD, CAT, T-AOC, and GSH-PX activity ( $p<0.01$ and $p<0.05$, respectively; Figure 8). Therefore, $18 \beta$-GA treatment inhibited MCT-induced oxidative stress. 


\section{A}
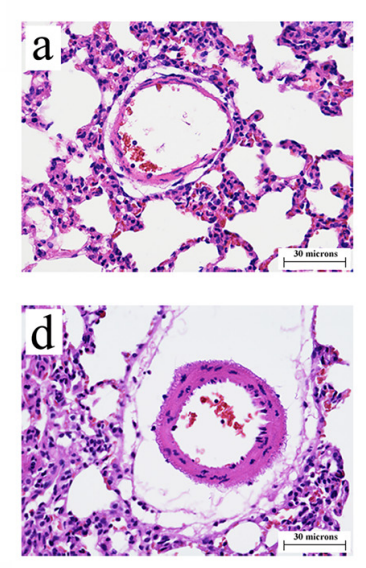

B
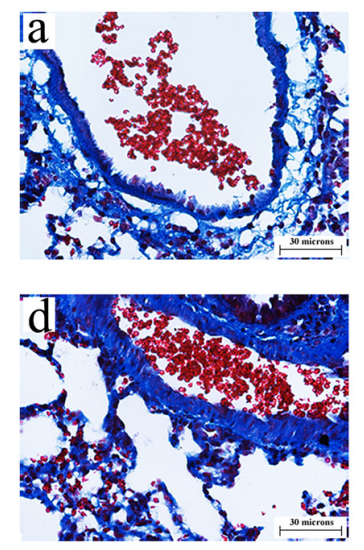

C

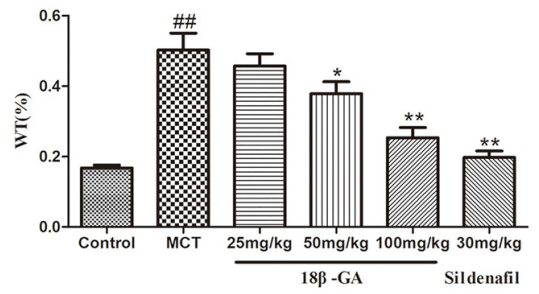

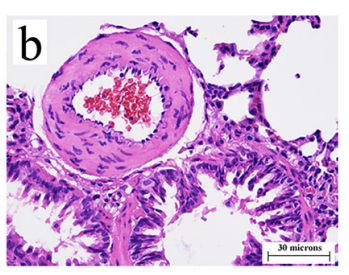
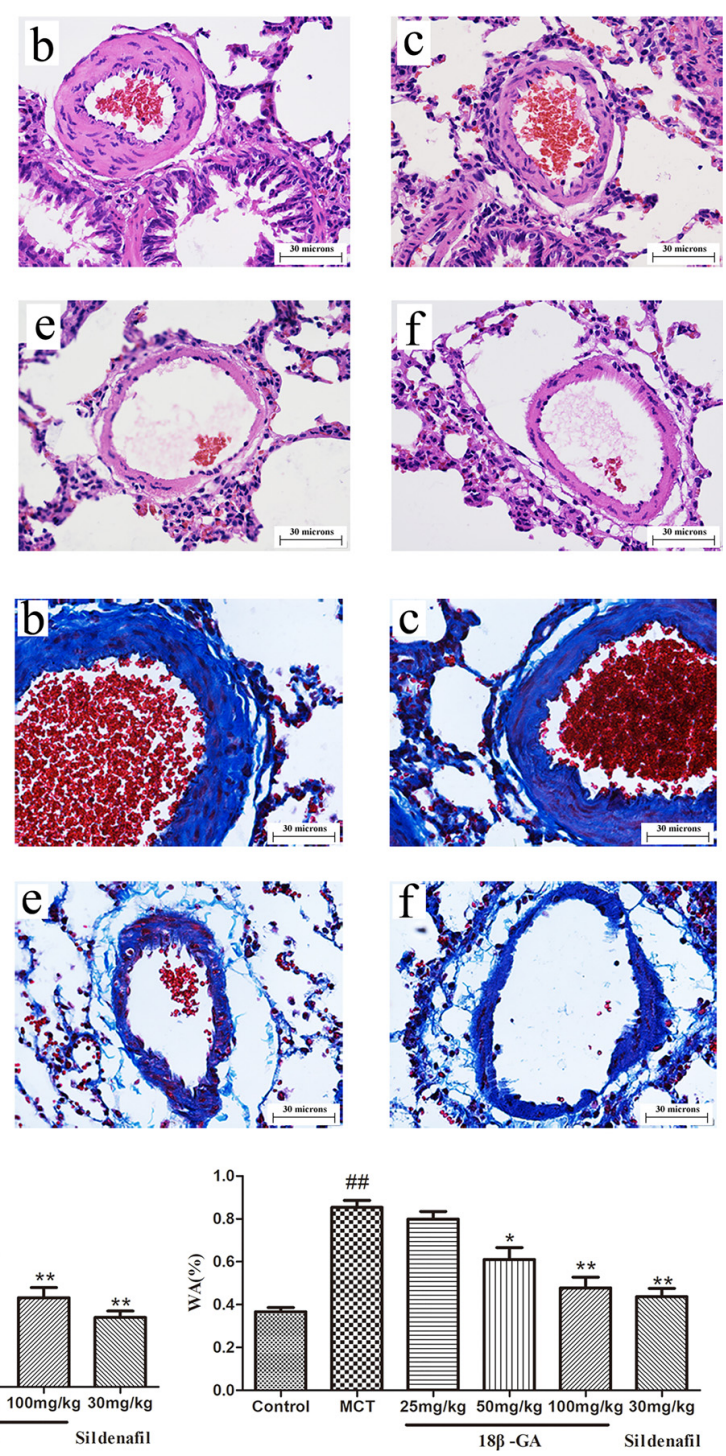

FIGURE 6 | Effect of 18ß-glycyrrhetinic acid on MCT-induced pulmonary vascular remodeling. Representative photomicrographs of pulmonary small arteries by H\&E

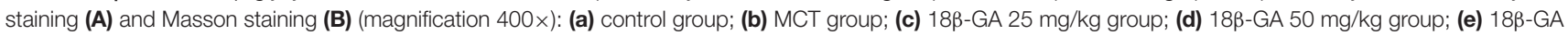
$100 \mathrm{mg} / \mathrm{kg}$ group; (f) Sildenafil $30 \mathrm{mg} / \mathrm{kg}$ group. (C) Left: Effect of $18 \beta-G A$ on WT\%, Right: Effect of $18 \beta-G A$ on WA\%. Data are expressed as mean \pm SEM ( $n=10$ ). $\# \#<0.01$ vs. control group, ${ }^{*} p<0.05,{ }^{* *} p<0.01$ vs. MCT group. MCT, monocrotaline; $18 \beta$-GA, $18 \beta$-glycyrrhetinic acid; WT\%, the ratio of the vascular walls thickness; WA\%, the ratio of the vascular wall area.

\section{Effects of 18 $\beta-$ GA on Nox2 and Nox4 Expression}

Based on the above studies, we found that $18 \beta$-GA can counteract the MCT-induced oxidation. To further investigate the link between $18 \beta$-GA and antioxidant effects, we examined the influence of $18 \beta-G A$ on the protein expression of Nox2 and Nox4, which could be related to antioxidant effects. The results indicated that the protein expression levels of Nox2 and Nox4 were up-regulated in the MCT-treated group compared with those in the control group $(p<0.01$ and $p<0.05$, Figure 9, respectively). 18 $\beta$-GA (100 mg/kg) treatment could reverse the increase in the Nox 2 and Nox4 expression levels in the lung tissue of the model rats, but their levels remained higher than those of the control group $(p<0.01$ and $p<0.05$, Figure 9, respectively). These results suggested that $18 \beta$-GA may against the progression of $\mathrm{PAH}$ at least partly via inhibition of the protein expression of Nox2 and Nox4.

\section{DISCUSSION}

This study reported for the first time that the intragastric administration of $18 \beta-\mathrm{GA}$ for 21 consecutive days exerted protective effects against MCT-induced $\mathrm{PAH}$ in rats. The 


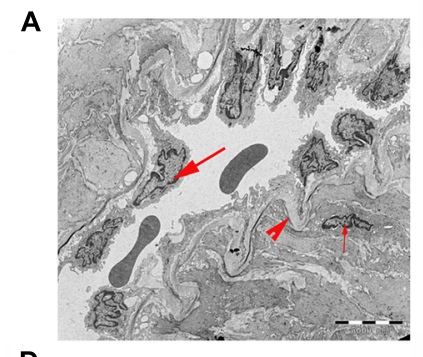

D

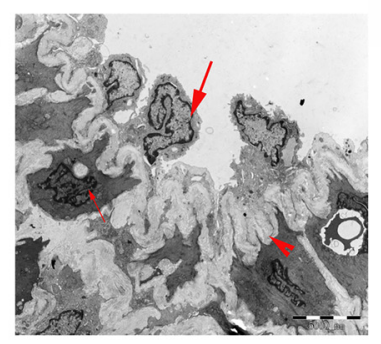

B

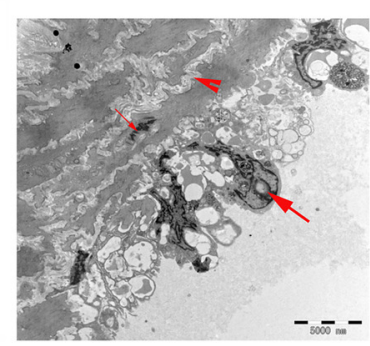

E

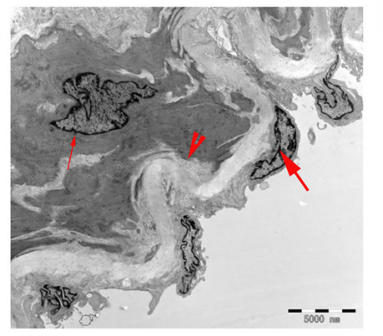

C

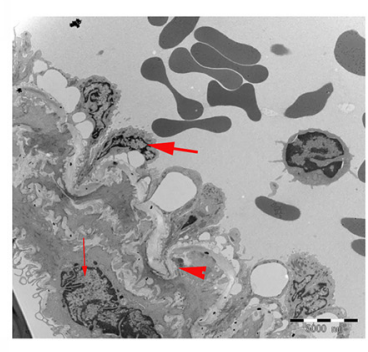

$\mathbf{F}$

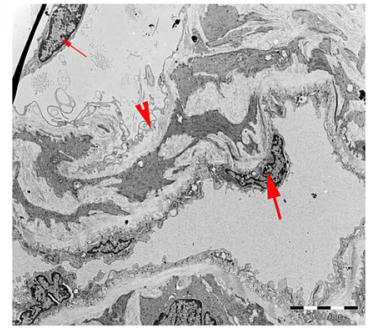

FIGURE 7 | Ultrathin cross-sections of the pulmonary vascular in rats were examined under transmission electron microscopy $(6,000 \times$ magnification;

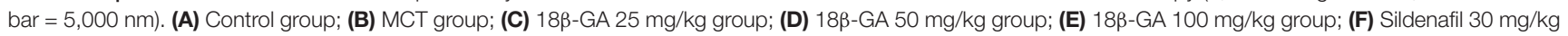

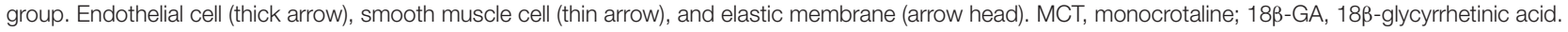

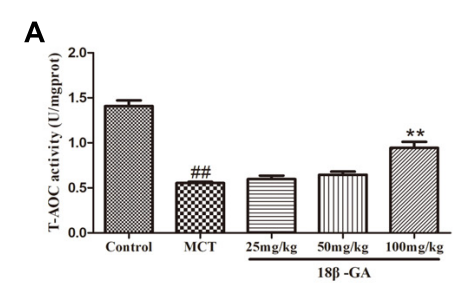

D
B

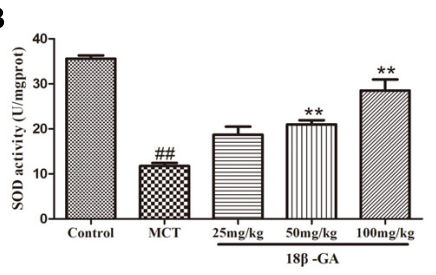

C

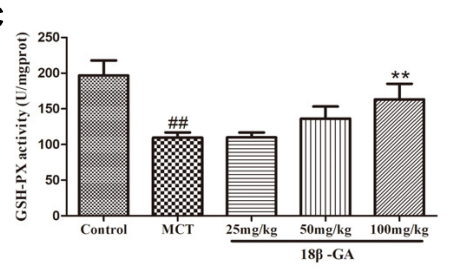

E
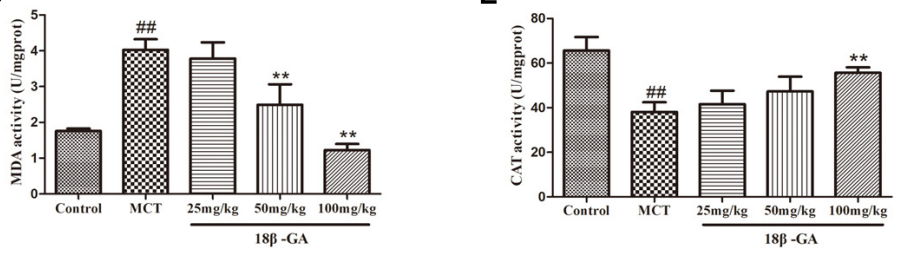

FIGURE 8 | Effect of 18ß-glycyrrhetinic acid on oxidative stress induced by MCT. (A) Effect of $18 \beta$-glycyrrhetinic acid on T-AOC expression. (B) Effect of

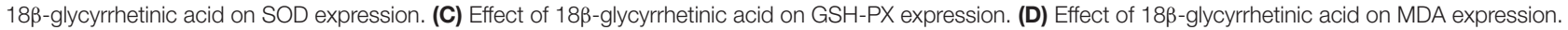
(E) Effect of $18 \beta$-glycyrrhetinic acid on CAT expression. Data are expressed as mean $\pm \mathrm{SEM}(n=10)$. ${ }^{\# \#} p<0.01 \mathrm{vs.} \mathrm{control}$ group, ${ }^{* *} p<0.01 \mathrm{vs.} \mathrm{MCT} \mathrm{group.}$ T-AOC, total antioxidant capacity; SOD, determine superoxide dismutase; GSH-PX, glutathione peroxidase; MDA, malonyldialdehyde; CAT, catalase; MCT,

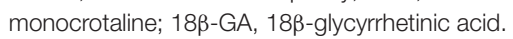

present study demonstrated that the survival rate of $\mathrm{PAH}$ model rats treated with $18 \beta-G A$ significantly improved. We observed that treatment with $18 \beta-G A$ attenuated lung and heart damage and inhibited the increase in MPAP, RVSP, and RVHI levels in the MCT-induced rat model. Histological verification showed that the pathological changes in the pulmonary vasculature induced by MCT were reversed. WA $\%$ and WT\% of the pulmonary artery improved, whereas the proliferation of pulmonary fibrous tissue decreased. Meanwhile, oxidative stress was inhibited in the rats with MCT-induced PAH. Western blot analysis results revealed that $18 \beta-\mathrm{GA}$ administration also down-regulated the protein overexpression of Nox 2 and Nox4 in the lungs. In summary, 18 $\beta$-GA provided a protective effect against MCT-induced PAH by inhibiting oxidative stress.

Pulmonary arterial hypertension, a pathological condition with low survival rates, is characterized by an increase in pulmonary arterial pressure, excessive pulmonary vascular remodeling, and subsequent right heart dysfunction (Haga et al., 2014; Lee et al., 2016). In this experiment, we used the rat model induced by MCT. When MCT was administered, its metabolites were distributed via pulmonary circulation in the body, where they could damage pulmonary vascular endothelial cells and cause vascular stenosis. The resistance 

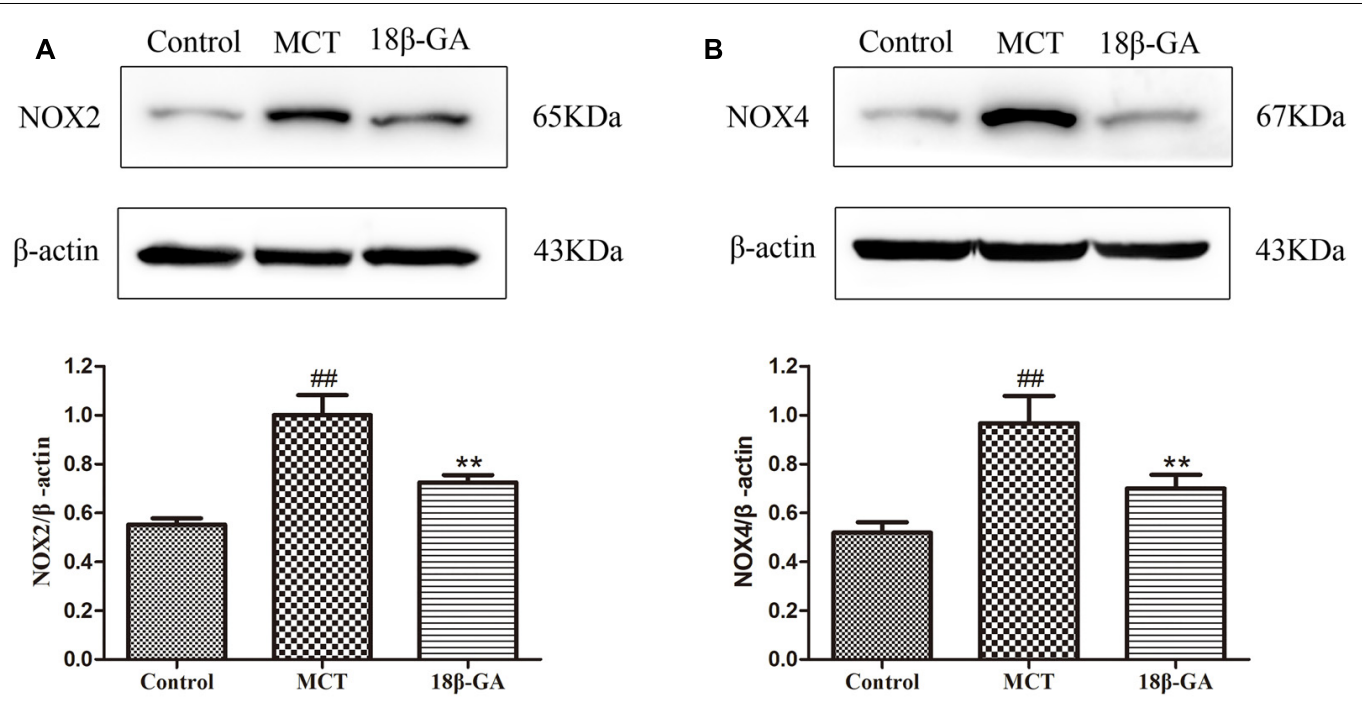

FIGURE 9 | Effects of 18ק-glycyrrhetinic acid on NOX-2 and NOX-4 expression. (A) Representative Western blot band of NOX-2 activation in the lung tissues. (B) Representative Western blot band of NOX-4 activation in the lung tissues. Data are expressed as mean $\pm \mathrm{SEM}(n=10)$. ${ }^{\#} p<0.01$ vs. control group, ${ }^{*} p<0.05$, ${ }^{* *} p<0.01$ vs. MCT group. NOX-2, nicotinamide adenine dinucleotide phosphate (NADPH) oxidase-2; NOX-4, nicotinamide adenine dinucleotide phosphate (NADPH) oxidase-4; MCT, monocrotaline; GA, 18ß-glycyrrhetinic acid.

in lung vasculature, pulmonary arterial wall remodeling, and right ventricular hypertrophy increased. Consequently, $\mathrm{PAH}$ developed. This model can mimic many characteristics of human PAH (Gomezarroyo et al., 2012; Wu et al., 2017b). The MCT-induced PAH model is also characterized by elevated levels of superoxide in the lungs and right ventricles (Singal et al., 2004; Kamezaki et al., 2008; Demarco et al., 2010). Thus, we chose the MCT-induced PAH model for our research.

$18 \beta-G A$ is a biologically active ingredient of licorice with an evident antioxidant effect and promising lung protective activity (Tzuchien et al., 2010; Huang et al., 2013; Hasan et al., 2014; Chen et al., 2017). In our pre-experiment, the optimal dosage range was 25-100 mg/kg (Oztanir et al., 2014; Mahmoud et al., 2017), and no significant toxicity was observed. On the basis of treatment strategies associated with clinical practices and our previous research experience, we used 18 $\beta$-GA after MCT was subcutaneously injected for 21 days and observed the survival rate of rats during the administration period (Mcmurtry et al., 2007; Wu et al., 2017a,b). A decline in the survival rate of PAH rat models was used as a marker of disease deterioration (Sun et al., 2017). In our experiment, the survival rate of $18 \beta$-GA-treated rats significantly improved compared with that in the MCT group. Therefore, $18 \beta$-GA could improve the quality of life of animals suffering from PAH.

Human pulmonary hypertension is characterized by abnormal pulmonary hemodynamic changes, including a marked increase in mPAP and RVSP and occurrence of RVH (Temple et al., 2014; Brewis et al., 2015). In animal models, the similar changes in mPAP, RVSP, and RVHI are often considered evidence of PAH development (Shi R. et al., 2017). Therefore, our study examined the effects of $18 \beta-\mathrm{GA}$ on hemodynamic parameters and RVHI. Our results suggested that $18 \beta-\mathrm{GA}$ therapy blocked the increase in mPAP, RVSP, and RVHI in a dosedependent manner. These findings indicated that $18 \beta-$ GA could prevent PAH.

Abnormal pulmonary vascular remodeling is considered the primary pathological feature of PAH (Bai et al., 2017), including pulmonary artery wall thickening and pulmonary artery stenosis. These changes can cause vasoconstriction resistance and increased cardiac after-load, eventually leading to PAH. H\&E staining and Masson trichrome staining were applied to measure the medial wall thickness of the pulmonary artery (WA\% and WT\%) and degree of pulmonary fibrosis and evaluate pulmonary vascular remodeling (Guignabert et al., 2013; Aiello et al., 2016; Lee et al., 2016). Transmission electron microscopy was performed to analyze the changes in the ultrastructure of the pulmonary artery. The pulmonary vascular medial wall thickness and vascular proliferation significantly increased in the MCT-treated groups, and this increase was also an indicator of PAH. Under the electron microscope, the smooth muscles of the pulmonary arterioles were hyperplastic, the collagen fibers of the adventitia were dense, and the endothelial cells were prominent in the model group. These features were effectively changed by the daily intragastric administration of $18 \beta$-GA, suggesting that $18 \beta$-GA elicited a protective effect against $\mathrm{PAH}$ by curbing pulmonary vascular remodeling.

Oxidative stress is a critical contributory element in various physiological and pathological processes and in the pathogenesis of PAH caused by MCT (Demarco et al., 2010). The pathological features of PAH mainly include pulmonary vasoconstriction and pulmonary artery wall remodeling (Bai et al., 2017). Oxidative stress has been described in the literature as a pathogenic mechanism of vascular remodeling observed in PAH (Barman et al., 2014). In the body, most of the vascular cells, including 
endothelial cells, smooth muscle cells, and adventitial cells, produce reactive oxygen species. Among the patients with vascular disease, the oxidative-antioxidative balance in blood vessel walls is impaired due to the increased reactive oxygen produced by these cells, thereby promoting vasoconstriction, smooth muscle cell proliferation, and vascular remodeling (Zhang et al., 2015). These findings suggested that limiting oxidative stress could help alleviate PAH. After reviewing many references, we found that oxidative stress is characterized by an increase in the production of oxidants (e.g., MDA) and a decrease in the concentrations of antioxidants and antioxidant enzymes (e.g., SOD, CAT, and GSH-PX) (Zimmermann et al., 2004). MDA is a toxic product of lipid peroxidation and a sensitive marker of oxidative stress (Chen et al., 2011). Highperformance antioxidant defense systems, including endogenous antioxidant enzymes (such as GSH-PX, SOD, CAT, and T-AOC), play an critical role in maintaining low oxidant concentrations and redox balance and in determining the active state that can reflect the degree of oxidative stress (Tabima et al., 2012). In our study, we examined the changes in SOD, CAT, MDA, T-AOC, and GSH-PX levels. Compared with the control group, MCT-treated rats presented significantly reduced SOD, CAT, T-AOC, and GSH-PX concentrations and increased MDA levels, but $18 \beta-G A$ administration reversed these changes.

Nox-derived products can trigger oxidative stress. In the vasculature, they can help maintain vascular tone and regulate important processes, such as cell growth, proliferation, differentiation, apoptosis, cell migration, and cytoskeletal organization (Wolin et al., 2005; Manea, 2010). The Nox family is composed of seven members; among them, Nox2 and Nox4 are the chief isomers distributed in the cardiovascular system. Nox2 and Nox4 are up-regulated in lung tissues from animal models and humans with PAH (Green et al., 2012; Barman et al., 2014), and they are likely involved in the development of increased pulmonary artery resistance and pressure (Liu et al., 2006; Demarco et al., 2010; Wu et al., 2017a). Nox2 plays a significant role in endothelial dysfunction in $\mathrm{PAH}$ ( $\mathrm{Li}$ et al., 2008; Liu et al., 2016). Nox4 is up-regulated in rats with MCTtreated and chronic hypoxia-induced PAH (Nisbet et al., 2009). Nox4 promotes the hypoxia-induced growth of HPASMCs, and silencing of Nox 4 expression by RNA interference can reduce the proliferation of human PASMCs and fibroblasts (Sanders and Hoidal, 2007). In this study, we investigated the protein expression of Nox 2 and Nox 4 by Western blot analysis. The results showed that the protein levels of Nox 2 and Nox 4 in the $18 \beta$-GA-treated group were obviously lower than those in the MCT-treated group. These experimental results indicated that $18 \beta$-GA could attenuate oxidative stress and reduce Nox 2 and Nox4 expression.

According to the control principle of the experimental program, the positive drug group is necessary to verify the rationality of our experimental program and the reliability of the protective effects of $18 \beta$-GA against PAH. Several studies have reported that sildenafil possesses a protective effect on MCT-induced PAH (Jasińskastroschein et al., 2014). The Food and Drug Administration also certified sildenafil for use in the treatment of PAH. Therefore, sildenafil was selected as a positive agent, and it exerted a protective effect on MCT-induced PAH as previously described in our study. There are some limitations in this study. The pathological mechanism of PAH is complex, such as and inflammation endothelial dysfunction. However, we have only studied the antioxidant effects of $18 \beta$-GA on $\mathrm{PAH}$, which is not comprehensive and in-depth. Therefore, we will thoroughly test the effects of $18 \beta-\mathrm{GA}$ on the inflammatory process in future studies.

\section{CONCLUSION}

In this study, we first demonstrated that $18 \beta-\mathrm{GA}$ administered to rats with $\mathrm{PAH}$ induced by MCT elicited a significant protective effect. 18 $\beta$-GA treatment improved hemodynamics and right ventricular hypertrophy and reduced cardiopulmonary injury. The protective effect of $18 \beta-\mathrm{GA}$ against $\mathrm{PAH}$ was related with the inhibition of oxidative stress and down-regulation of Nox2 and Nox4 levels. Therefore, the experimental data supported the view that $18 \beta-\mathrm{GA}$ is beneficial for the treatment of PAH.

\section{ETHICS STATEMENT}

This study was carried out in accordance with the recommendations of the Institutional Animal Care and Use Committee of Ningxia Medical University. The protocol was approved by the Institutional Animal Care and Use Committee of Ningxia Medical University.

\section{AUTHOR CONTRIBUTIONS}

ZC, NL, J-LW, and Y-JH performed data curation. RZ and Q-BX conducted project administration. LY, PM, WZ, LB, and $\mathrm{XM}$ carried through supervision. MZ processed writing-original draft. RZ and MZ executed writing-review and editing. All authors commented on and approved the final manuscript.

\section{FUNDING}

This project was supported by 2017 Ningxia Hui Autonomous Region Science and Technology innovation leader training project (Grant No. KJT2017005); 2017 Ningxia medical university youth backbone talent cultivation selected project; Development of Glycyrrhetinic Acid on Pulmonary Hypertension (Ningxia Key R\&D Program Project Foreign Science and Technology Cooperation Special, Grant No. 2018BFH03019); Key Research and Development Plan of Ningxia Hui Autonomous Region in 2019 (special cooperation for foreign cooperation).

\section{ACKNOWLEDGMENTS}

We are indebted to the staff in the Animal Center and the Science and Technology Centre who provided assistance in the study. 


\section{REFERENCES}

Aiello, R. J., Bourassa, P. A., Zhang, Q., Dubins, J., Goldberg, D. R., Lombaert, S. D., et al. (2016). Tryptophan hydroxylase 1 (TPH1) inhibition impacts pulmonary vascular remodeling in two rat models of pulmonary hypertension. J. Pharmacol. Exp. Ther. 360, 267-279. doi: 10.1124/jpet.116.237933

Bai, Y., Li, Z. X., Zhao, Y. T., Liu, M., Wang, Y., Lian, G. C., et al. (2017). PCPA protects against monocrotaline-induced pulmonary arterial remodeling in rats: potential roles of connective tissue growth factor. Oncotarget 8, 111642-111655. doi: 10.18632/oncotarget.22882

Barman, S. A., Chen, F., Su, Y., Dimitropoulou, C., Wang, Y., Catravas, J. D., et al. (2014). NADPH oxidase 4 is expressed in pulmonary artery adventitia and contributes to hypertensive vascular remodeling. Arterioscler. Thromb. Vasc. Biol. 34:1704. doi: 10.1161/ATVBAHA.114.303848

Benza, R. L., Miller, D. P., Barst, R. J., Badesch, D. B., Frost, A. E., and Mcgoon, M. D. (2012). An evaluation of long-term survival from time of diagnosis in pulmonary arterial hypertension from the reveal registry. Chest 142, 448-456. doi: 10.1378/chest.11-1460

Brewis, M. J., Church, A. C., Johnson, M. K., and Peacock, A. J. (2015). Severe pulmonary hypertension in lung disease: phenotypes and response to treatment. Eur. Respir. J. 46, 1378-1389. doi: 10.1183/13993003.02307-2014

Chen, H., Yoshioka, H., Kim, G. S., Jung, J. E., Okami, N., Sakata, H., et al. (2011). Oxidative stress in ischemic brain damage: mechanisms of cell death and potential molecular targets for neuroprotection. Antioxid. Redox. Signal. 14, 1505-1517. doi: 10.1089/ars.2010.3576

Chen, J., Zhang, W., Zhang, L., Zhang, J., Chen, X., Yang, M., et al. (2017). Glycyrrhetinic acid alleviates radiation-induced lung injury in mice. J. Radiat. Res. 58, 41-47. doi: 10.1093/jrr/rrw091

Chung, W. T., Lee, S. H., Kim, J. D., Sung, N. S., Hwang, B., Lee, S. Y., et al. (2001). Effect of the extracts from Glycyrrhiza uralensis fisch on the growth characteristics of human cell lines: anti-tumor and immune activation activities. Cytotechnology 37:55. doi: 10.1023/A:1016111713056

Demarco, V. G., Whaley-Connell, A. T., Sowers, J. R., Habibi, J., and Dellsperger, K. C. (2010). Contribution of oxidative stress to pulmonary arterial hypertension. World J. Cardiol. 2, 316-324. doi: 10.4330/wjc.v2.i10.316

Galiè, N., and Manes, A. (2013). New treatment strategies for pulmonary arterial hypertension: hopes or hypes? J. Am. Coll. Cardiol. 62, 1101-1102. doi: 10.1016/ j.jacc.2013.06.032

Gao, H., Cheng, Y., Zong, L., Huang, L., Qiao, C., Li, W., et al. (2017). Aspirin attenuates monocrotaline-induced pulmonary arterial hypertension in rats by suppressing the ERK/MAPK pathway. Clin. Exp. Hypertens. 39, 34-41. doi: 10.1080/10641963.2016.1210620

Gomezarroyo, J. G., Farkas, L., Alhussaini, A. A., Farkas, D., Kraskauskas, D., Voelkel, N. F., et al. (2012). The monocrotaline model of pulmonary hypertension in perspective. Am. J. Physiol. Lung. Cell Mol. Physiol. 302, 363-369. doi: 10.1152/ajplung.00212.2011

Green, D. E., Murphy, T. C., Kang, B. Y., Kleinhenz, J. M., Szyndralewiez, C., Page, P., et al. (2012). The Nox4 inhibitor GKT137831 attenuates hypoxiainduced pulmonary vascular cell proliferation. Am. J. Respir. Cell Mol. Biol. 47:718. doi: 10.1165/rcmb.2011-0418OC

Guignabert, C., Tu, L., Hiress, M. L., Ricard, N., Sattler, C., Seferian, A., et al. (2013). Pathogenesis of pulmonary arterial hypertension: lessons from cancer. Eur. Respir. Rev. 22, 543-551. doi: 10.1183/09059180. 00007513

Haga, S., Tsuchiya, H., Hirai, T., Hamano, T., Mimori, A., and Ishizaka, Y. (2014). A novel ACE2 activator reduces monocrotaline-induced pulmonary hypertension by suppressing the JAK/STAT and TGF- $\beta$ cascades with restored caveolin-1 expression. Exp. Lung Res. 41, 21-31. doi: 10.3109/01902148.2014. 959141

Hasan, S. K., Khan, R., Ali, N., Khan, A. Q., Rehman, M. U., Tahir, M., et al. (2014). 18- $\beta$ Glycyrrhetinic acid alleviates 2-acetylaminofluoreneinduced hepatotoxicity in Wistar rats: role in hyperproliferation, inflammation and oxidative stress. Hum. Exp. Toxicol. 34, 628-641. doi: 10.1177/ 0960327114554045

Huang, R. Y., Chu, Y. L., Huang, Q. C., Chen, X. M., Jiang, Z. B., Zhang, X., et al. (2013). 18 $\beta$-Glycyrrhetinic acid suppresses cell proliferation through inhibiting thromboxane synthase in non-small cell lung cancer. PLoS One 9:e93690. doi: 10.1371/journal.pone.0093690
Humbert, M., Sitbon, O., Chaouat, A., Bertocchi, M., Habib, G., Gressin, V., et al. (2010). Survival in patients with idiopathic, familial, and anorexigen-associated pulmonary arterial hypertension in the modern management era. Circulation 122:156. doi: 10.1161/CIRCULATIONAHA.109.911818

Jasińskastroschein, M., Owczarek, J., Wesołowska, A., and Orszulakmichalak, D. (2014). Rosuvastatin, sildenafil and their combination in monocrotalineinduced pulmonary hypertension in rat. Acta Pharm. 64, 345-353. doi: 10.2478/ acph-2014-0029

Kamezaki, F., Tasaki, H., Yamashita, K., Tsutsui, M., Koide, S., Nakata, S., et al. (2008). Gene transfer of extracellular superoxide dismutase ameliorates pulmonary hypertension in rats. Am. J. Respir. Crit. Care Med. 177, 219-226. doi: 10.1164/rccm.200702-264OC

Karpuz, D., Hallioglu, O., Buyukakilli, B., Gurgul, S., Balli, E., Ozeren, M., et al. (2017). Clinical and histopathological relationship of sildenafil and bosentan treatments in rats with monocrotaline induced pulmonary hypertension. Bratisl. Lek. Listy 118, 544-551. doi: 10.4149/BLL_2017_104

Lan, T. H., Chen, X. L., Wu, Y. S., Qiu, H. L., Li, J. Z., Ruan, X. M., et al. (2018). 3,7-Bis(2-hydroxyethyl)icaritin, a potent inhibitor of phosphodiesterase5 , prevents monocrotaline-induced pulmonary arterial hypertension via NO/cGMP activation in rats. Eur. J. Pharmacol. 829, 102-111. doi: 10.1016/j. ejphar.2018.04.011

Lee, J. H., Park, B. K., Oh, K. S., Yi, K. Y., Lim, C. J., Seo, H. W., et al. (2016). A urotensin II receptor antagonist, KR36676, decreases vascular remodeling and inflammation in experimental pulmonary hypertension. Int. Immunopharmacol. 40, 196-202. doi: 10.1016/j.intimp.2016.09.002

Li, S., Tabar, S. S., Malec, V., Eul, B. G., Klepetko, W., Weissmann, N., et al. (2008). NOX4 regulates ROS levels under normoxic and hypoxic conditions, triggers proliferation, and inhibits apoptosis in pulmonary artery adventitial fibroblasts. Antioxid. Redox Signal. 10, 1687-1698. doi: 10.1089/ars.2008.2035

Liu, B., Ren, K. D., Peng, J. J., Li, T., Luo, X. J., Fan, C., et al. (2016). Suppression of NADPH oxidase attenuates hypoxia-induced dysfunctions of endothelial progenitor cells. Biochem. Biophys. Res. Commun. 482, 1080-1087. doi: 10.1016/ j.bbrc.2016.11.161

Liu, J. Q., Zelko, I. N., Erbynn, E. M., Sham, J. S., and Folz, R. J. (2006). Hypoxic pulmonary hypertension: role of superoxide and NADPH oxidase (gp91phox). Am. J. Physiol. Lung Cell. Mol. Physiol. 290, L2-L10. doi: 10.1152/ajplung.00135. 2005

Mahmoud, A. M., Hussein, O. E., Hozayen, W. G., and Abd El-Twab, S. M. (2017). Methotrexate hepatotoxicity is associated with oxidative stress, and downregulation of PPAR $\gamma$ and Nrf2: protective effect of $18 \beta$-Glycyrrhetinic acid. Chem. Biol. Interact. 270, 59-72. doi: 10.1016/j.cbi.2017.04.009

Manea, A. (2010). NADPH oxidase-derived reactive oxygen species: involvement in vascular physiology and pathology. Cell Tissue Res. 342, 325-339. doi: 10. 1007/s00441-010-1060-y

Mcmurtry, M. S., Bonnet, S., Michelakis, E. D., Bonnet, S., Haromy, A., and Archer, S. L. (2007). Statin therapy, alone or with rapamycin, does not reverse monocrotaline pulmonary arterial hypertension: the rapamcyin-atorvastatinsimvastatin study. Am. J. Physiol. Lung Cell. Mol. Physiol. 293, L933-L940. doi: 10.1152/ajplung.00310.2006

Nisbet, R. E., Graves, A. S., Kleinhenz, D. J., Rupnow, H. L., Reed, A. L., Fan, T. H. M., et al. (2009). The role of NADPH oxidase in chronic intermittent hypoxia-induced pulmonary hypertension in mice. Am. J. Respir. Cell Mol. Biol. 40, 601-609. doi: 10.1165/2008-0145OC

Oztanir, M. N., Ciftci, O., Cetin, A., Durak, M. A., Basak, N., and Akyuva, Y. (2014). The beneficial effects of $18 \beta$-glycyrrhetinic acid following oxidative and neuronal damage in brain tissue caused by global cerebral ischemia/reperfusion in a C57BL/J6 mouse model. Neurol. Sci. 35, 1221-1228. doi: 10.1007/s10072014-1685-9

Sanders, K. A., and Hoidal, J. R. (2007). The NOX on pulmonary hypertension. Circ. Res. 101, 224-226. doi: 10.1161/CIRCRESAHA.107.158246

Shi, G. J., Zheng, J., Wu, J., Qiao, H. Q., Chang, Q., Niu, Y., et al. (2017). Beneficial effects of Lycium barbarum polysaccharide on spermatogenesis by improving antioxidant activity and inhibiting apoptosis in streptozotocin-induced diabetic male mice. Food Funct. 8, 1215-1226. doi: 10.1039/c6fo01575a

Shi, R., Wei, Z., Zhu, D., Fu, N., Chang, W., Yin, S., et al. (2017). Baicalein attenuates monocrotaline-induced pulmonary arterial hypertension by inhibiting vascular remodeling in rats. Pulm. Pharmacol. Ther. 48, 124-135. doi: 10.1016/j.pupt.2017.11.003 
Singal, P. K., Farahmand, F., and Hill, M. F. (2004). Antioxidant and oxidative stress changes in experimental cor pulmonale. Mol. Cell. Biochem. 260, 21-29. doi: 10.1023/B:MCBI.0000026047.48534.50

Sun, L. Y., Cai, Z. Y., Pu, J., Li, J., Shen, J. Y., Yang, C. D., et al. (2017). 5-Aminosalicylic acid attenuates monocrotaline-induced pulmonary arterial hypertension in rats by increasing the expression of Nur77. Inflammation 40, 1-12. doi: 10.1007/s10753-017-0525-5

Tabima, D. M., Frizzell, S., and Gladwin, M. T. (2012). Reactive oxygen and nitrogen species in pulmonary hypertension. Free Radic. Biol. Med. 52, 1970 1986. doi: 10.1016/j.freeradbiomed.2012.02.041

Temple, I. P., Monfredi, O., Quigley, G., Schneider, H., Zi, M., Cartwright, E. J., et al. (2014). Macitentan treatment retards the progression of established pulmonary arterial hypertension in an animal model. Int. J. Cardiol. 177, 423-428. doi: 10.1016/j.ijcard.2014.09.005

Thenappan, T., Shah, S. J., Rich, S., Tian, L., Archer, S. L., and Gombergmaitland, M. (2010). Survival in pulmonary arterial hypertension: a reappraisal of the NIH risk stratification equation. Eur. Respir. J. 35, 1079-1087. doi: 10.1183/09031936.00072709

Tzuchien, K., Minghuan, S., and Gowchin, Y. (2010). Glycyrrhizic acid and $18 \beta$-glycyrrhetinic acid inhibit inflammation via PI3K/Akt/GSK3 $\beta$ signaling and glucocorticoid receptor activation. J. Agric. Food Chem. 58, 8623-8629. doi: $10.1021 /$ jf101841r

Wedgwood, S., Lakshminrusimha, S., Czech, L., Schumacker, P. T., and Steinhorn, R. H. (2013). Increased p22phox/Nox4 expression is involved in remodeling through hydrogen peroxide signaling in experimental persistent pulmonary hypertension of the newborn. Antioxid. Redox Signal. 18, 1765-1776. doi: 10. 1089/ars.2012.4766

Wolin, M. S., Ahmad, M., and Gupte, S. A. (2005). The sources of oxidative stress in the vessel wall. Kidney Int. 67, 1659-1661. doi: 10.1111/j.1523-1755.2005. 00257.x

Wu, F., Hao, Y., Yang, J., Yao, W., Xu, Y., Yan, L., et al. (2017a). Protective effects of aloperine on monocrotaline-induced pulmonary hypertension in rats. Biomed. Pharmacother. 89, 632-641. doi: 10.1016/j.biopha.2017. 02.033

Wu, F., Yao, W., Yang, J., Zhang, M., Xu, Y., Hao, Y., et al. (2017b). Protective effects of aloperin on monocroline-induced pulmonary hypertension via regulation of Rho A/Rho kinsase pathway in rats. Biomed. Pharmacother. 95, 1161-1168. doi: 10.1016/j.biopha.2017.08.126

Wu, Y., Adi, D., Long, M., Wang, J., Liu, F., Gai, M. T., et al. (2016). 4-Phenylbutyric acid induces protection against pulmonary arterial hypertension in rats. PLoS One 11:e0157538. doi: 10.1371/journal.pone.0157538

Yang, N., Patil, S., Zhuge, J., Wen, M. C., Bolleddula, J., Doddaga, S., et al. (2014). Glycyrrhiza uralensis flavonoids present in anti-asthma formula, ASHMI, inhibit memory Th2 responses in vitro and in vivo. Phytother. Res. 27, 13811391. doi: $10.1002 /$ ptr.4862

Young, P. S., Jin, K. S., Sung, L. S., Jin-Kyu, K., Won, L. K., and Yoon, P. J. H. (2016). Licoricidin, an active compound in the hexane/ethanol extract of Glycyrrhiza uralensis, inhibits lung metastasis of 4T1 murine mammary carcinoma cells. Int. J. Mol. Sci. 17:934. doi: 10.3390/ijms17060934

Zhang, M. T., Wang, B., Jia, Y. N., Liu, N., Ma, P. S., Gong, S. S., et al. (2017). Neuroprotective effect of liquiritin against neuropathic pain induced by chronic constriction injury of the sciatic nerve in mice. Biomed. Pharmacother. 95, 186-198. doi: 10.1016/j.biopha.2017.07.167

Zhang, S., Yang, T., Xu, X., Wang, M., Zhong, L., Yang, Y., et al. (2015). Oxidative stress and nitric oxide signaling related biomarkers in patients with pulmonary hypertension: a case control study. BMC Pulm. Med. 15:50. doi: 10.1186/ s12890-015-0045-8

Zimmermann, C., Winnefeld, K., Streck, S., Roskos, M., and Haberl, R. L. (2004). Antioxidant status in acute stroke patients and patients at stroke risk. Eur. Neurol. 51, 157-161. doi: 10.1159/000077662

Conflict of Interest Statement: The authors declare that the research was conducted in the absence of any commercial or financial relationships that could be construed as a potential conflict of interest.

Copyright (c) 2019 Zhang, Chang, Zhao, Zhang, Hao, Yan, Liu, Wang, Bo, Ma, Zhou, Ma, Xu and Zhou. This is an open-access article distributed under the terms of the Creative Commons Attribution License (CC BY). The use, distribution or reproduction in other forums is permitted, provided the original author $(s)$ and the copyright owner(s) are credited and that the original publication in this journal is cited, in accordance with accepted academic practice. No use, distribution or reproduction is permitted which does not comply with these terms. 